\title{
Energy, Entropy and the Ricci Flow
}

\author{
Joseph Samuel and Sutirtha Roy Chowdhury \\ Raman Research Institute, Bangalore-560 080
}

(Dated: October 25, 2018)

\begin{abstract}
The Ricci flow is a heat equation for metrics, which has recently been used to study the topology of closed three manifolds. In this paper we apply Ricci flow techniques to general relativity. We view a three dimensional asymptotically flat Riemannian metric as a time symmetric initial data set for Einstein's equations. We study the evolution of the area $\mathcal{A}$ and Hawking mass $\mathcal{M}_{H}$ of a two dimensional closed surface under the Ricci flow. The physical relevance of our study derives from the fact that, in general relativity the area of apparent horizons is related to black hole entropy and the Hawking mass of an asymptotic round 2-sphere is the ADM energy. We begin by considering the special case of spherical symmetry to develop a physical feel for the geometric quantities involved. We then consider a general asymptotically flat Riemannian metric and derive an inequality $\frac{d}{d \tau} \mathcal{A}^{3 / 2} \leq-24 \pi^{3 / 2} \mathcal{M}_{H}$ which relates the evolution of the area of a closed surface $S$ to its Hawking mass. We suggest that there may be a maximum principle which governs the long term existence of the asymptotically flat Ricci flow.
\end{abstract}

PACS numbers: 02.40.-k,04.70.Dy 


\section{INTRODUCTION}

The Ricci flow [1, 2, 3, 4] has been used by mathematicians to understand the topology of three manifolds. It appears likely that these mathematical developments will also be useful in physics in the study of geometric theories like general relativity. Several papers [5, 6, 7, 8, 9, 10, 11] have appeared dealing with physical applications of the Ricci flow. The Ricci flow (RF) is a (degenerate) parabolic differential equation, and is very similar to the heat equation. In a previous paper [12] we explored an analogy between the Ricci flow and thermodynamics. The analogy is based on the observation that the Ricci flow (like the heat equation) loses memory of initial conditions, just as a physical system loses memory of its initial state as it approaches thermal equilibrium. As was noticed there, a slight modification of the Ricci flow yields Schwarzschild space as a fixed point. In this paper we look at the unmodified Ricci flow to see how some physically interesting quantities evolve with the flow. Energy and entropy are quantities of physical interest from the thermodynamic point of view. In general relativity these quantities take on a purely differential geometric meaning: the entropy is related to the area of black hole horizons and the energy to the ADM mass at infinity. We investigate the the evolution of these quantities under the Ricci flow and derive some inequalities relating them.

The subject of entropy bounds [13, 14] has been of wide and sometimes controversial [15] interest to physicists. It is believed that such bounds contain clues towards quantum gravity. Entropy bounds motivate ideas like "the holographic hypothesis". There is a geometric entropy bound proposed by Penrose, which connects two geometrical quantities: the area of the outermost horizon and the ADM mass. In the seventies, Roger Penrose[16], in an attempt to test the idea of cosmic censorship (which is part of the "establishment view" of gravitational collapse), used physical arguments to deduce from cosmic censorship an inequality relating the ADM mass of an initial data set for GR and the area of its outermost apparent horizon: $M_{A D M} \geq \sqrt{16 \pi \mathcal{A}}$. This inequality, which is saturated by the Schwarzschild space, has a clear thermodynamic interpretation. It states that Schwarzschild spacetime maximises geometric entropy for a given energy. Maximising entropy for fixed energy is a property which characterises a thermal state in statistical mechanics. The thermal character of Hawking radiation from a Schwarzschild black hole is entirely consistent with this interpretation. Penrose's inequality appears to capture something deep about general 
relativity, with a thermodynamic statement expressed in geometric terms. In this respect, it is similar to the the area theorem[17], which represents the second law of thermodynamics in geometric form.

A counterexample to Penrose's inequality would imply a flaw in the establishment view. No counterexample has so far been found. Special cases of Penrose's inequality have been proved[18, 19, 20] using geometric flow techniques. Jang and Wald [21] showed that if one assumes the existence of the inverse mean curvature (IMC) flow, (a particular diffeomorphism of a spatial slice), one could address Penrose's inequality in the special case of time symmetric initial data. Their work was based on the monotonicity of the Hawking mass under the inverse mean curvature flow, which follows from earlier work by Geroch[22]. This suggests a proof of the Penrose inequality [21]. The only gap in this proof is the existence of the IMC flow. This gap was filled by Huisken and Ilmanen [20] who showed that the Hawking mass remained monotonic under a discontinuous version of the IMC flow.

The motivation behind the present work is to explore whether the Ricci flow could lead to a new proof of the time symmetric Penrose inequality. The general Penrose inequality is still an open conjecture and new lines of attack (even in the time symmetric case) are certainly of interest. Is it possible that a smooth combination of the Ricci flow plus diffeomorphisms exists so that the evolution of Hawking mass is monotonic? The hope underlying the use of the RF is that the discontinuities (like those of the IMC flow) will be smoothed out by the RF term. There are known examples in fluid mechanics where discontinuities of inviscid flow are smoothed out by viscous effects. With this motivation of developing a Ricci flow approach to the Penrose inequality, we have made a beginning in this paper by studying the evolution of area and Hawking mass under the Ricci flow.

In section II we give a brief review of the Ricci flow. For a more complete and rigorous account the reader is referred to the mathematical literature[23, 24]. Section III lists the geometrical quantities of physical interest in this paper. Section IV paves the way for the general treament by treating the special case of spherical symmetry. This special case is useful since one can explicitly work out geometrical quantities of interest and develop a physical feel for them. Spherical symmetry is a good source of physical examples and counterexamples which guide the general study. In Section V we give up spherical symmetry and treat the evolution of area of a closed surface under the Ricci flow and derive an inequality relating the rate of change of area and the Hawking mass. Section VI treats the evolution of 
Hawking mass under Ricci flow and puts forward a conjectured maximum principle which governs the long time behaviour of the flow. Section VII is a concluding discussion. Our metric conventions are from Ref.[25].

\section{THE RICCI FLOW}

Let $\left(\Sigma, h_{a b}\right)$ be an asymptotically flat, three dimensional Riemannian manifold. ( $a, b$ run over 1,2,3. We restrict our discussion to three dimensional manifolds.) Our interest is in asymptotically flat spaces since we are interested in the energy and entropy of black holes. The definitions of energy, entropy and black holes all need an asymptotic region. The total energy or ADM mass of an initial data set is only well defined if an asymptotic structure (either flat or AdS) is fixed. Black holes are defined as regions of spacetime from which escape to infinity is impossible and thus refer to an asymptotic structure. We require that the metric tend to a fixed flat metric $\delta_{a b}$ at infinity $h_{a b} \rightarrow \delta_{a b}+O(1 / r)$. Given an initial metric $h_{a b}$, the Ricci flow evolves the metric according to its Ricci tensor. The evolution parameter is $\tau$ and the family of metrics on $\left(\Sigma, h_{a b}(\tau)\right)$ satisfies the Ricci flow equation

$$
\frac{\partial h_{a b}}{\partial \tau}=-2 R_{a b} .
$$

In the neighborhood of a point $p \in \Sigma$, we can introduce a Riemann normal co-ordinate system and then the form of (11) becomes parabolic ( $\nabla^{2}$ is the Laplacian in local co-ordinates)

$$
\frac{\partial h_{a b}}{\partial \tau}=\nabla^{2} h_{a b}
$$

and looks like a heat equation for the metric coefficients. However, in a general co-ordinate system, the PDE (1) is a degenerate parabolic equation, because of its diffeomorphism invariance.

More generally, we will be interested in the Ricci flow modified by a diffeomorphism

$$
\frac{\partial h_{a b}}{\partial \tau}=-2 R_{a b}+D_{a} \xi_{b}+D_{b} \xi_{a},
$$

where $\xi^{a}$ is any vector field on $\Sigma$ which vanishes at infinity. Calculationally, it is convenient to consider the two terms separately, defining a pure Ricci flow (1) and a pure diffeo

$$
\frac{\partial h_{a b}}{\partial \tau}=D_{a} \xi_{b}+D_{b} \xi_{a} .
$$


In the mathematical literature on Ricci flows (which deals with compact spaces) the term $\lambda h_{a b}$ is sometimes added to the RHS of (11) to define a "normalised Ricci flow". Such a term is inadmissible in the present physical context as it would rescale the metric at infinity and violate our asymptotic requirement that the metric tends to a fixed flat metric at infinity.

In the standard initial value formulation [25] of general relativity, the basic variables are the induced metric $h_{a b}$ on a spatial slice $\Sigma$ in the space-time manifold $M, \Sigma \in M$ and the extrinsic curvature $k_{a b}$ of $\Sigma$. We also use the notation $k=h^{a b} k_{a b}$. These variables are subject to constraints:

$$
D_{b}\left(k^{a b}-h^{a b} k\right)=8 \pi j^{b}
$$

and

$$
R+k^{2}-k_{a b} k^{a b}=16 \pi \rho,
$$

where $j^{b}$ is the matter current and $\rho$ is the matter density. The matter is required to satisfy "energy conditions", the dominant, weak or strong energy condition.

A three dimensional manifold $\left(\Sigma, h_{a b}\right)$ can be viewed as a time symmetric intitial data set for Einstein's equations. By "time symmetric" we mean that the extrinsic curvature $k_{a b}$ of $\Sigma$ has been set to zero, so that $\left(\Sigma, h_{a b}\right)$ is totally geodesic. (This is similar to choosing initial data in classical mechanics so that all the momenta vanish. Dropping $\mathrm{N}$ particles from rest is an example.) With $k_{a b}$ (and the matter current $j^{a}$ ) set to zero, the diffeomorphism constraint (5) is automatically satisfied and the Hamiltonian constraint reduces to $R=16 \pi \rho$. A physically important constraint on initial data for general relativity is that the data are subject to an energy condition. The dominant, weak and strong energy conditions all imply the local energy condition, which states that the local energy density is non-negative. This translates into the geometrical statement that the scalar curvature $R$ of $h_{a b}$ be non-negative. The Ricci flow has the appealing property that it preserves the non-negativity of scalar curvature[24]. This follows from the "maximum principle" for the scalar curvature. Under Ricci flow(3), the scalar curvature satisfies the non linear heat type equation

$$
\frac{\partial R}{\partial \tau}=\nabla^{2} R+2 R^{a b} R_{a b}+\mathcal{L}_{\xi} R
$$

which implies that $\frac{\partial R}{\partial \tau}$ is positive at a minimum of $R$. Eq.(3) provides us with a flow on the space of initial data to Einstein's equations which remains within physically allowed (non-negative scalar curvature) data. 


\section{GEOMETRIC QUANTITIES OF INTEREST}

Let $S$ be a closed surface in $\Sigma, \gamma_{i j},(i, j=1,2)$ the pull back or induced metric on $S$, $\mathcal{R}$ the scalar curvature of $\left(S, \gamma_{i j}\right)$ and $K$ the trace of its extrinsic curvature. We will be interested in the evolution of some geometric properties of $S$ under the Ricci flow. These are the area of $S$,

$$
\mathcal{A}(S)=\int_{S} d A=\int_{S} d^{2} x \sqrt{\gamma}
$$

and the Hawking mass of $S$

$$
\mathcal{M}_{H}(S)=\frac{\sqrt{\mathcal{A}(S)}}{64 \pi^{3 / 2}} \int_{S} d A\left(2 \mathcal{R}-K^{2}\right) .
$$

Our interest in these quantities stems from their physical significance. The area of apparent horizons is related to the entropy of Black Holes and the Hawking Mass is related to the Energy. The Hawking Mass of a surface $S$ can be physically interpreted as the mass contained within the surface $S$. While there are some problems with this interpretation (positivity is not always assured), the Hawking mass is an useful notion[21, 22] of quasilocal mass. It vanishes in the limit that $S$ shrinks to a point and becomes the ADM energy for a round sphere at infinity. In fact, the supremum of $\mathcal{M}(S)$ over $S$ is the ADM mass as one can see from [21, 22]. Unlike the ADM energy, which is only well-defined for asymptotic spheres, the Hawking mass is defined for any closed surface $S$. Under the RF, a surface $S$, which is initially asymptotic may shrink into the interior of $\Sigma$. For this reason Hawking mass is a more convenient object to study than the ADM mass.

Rather than the Hawking mass, it is more convenient to deal with the related dimensionless quantity the "compactness" of $S$

$$
\mathcal{C}(S)=\int_{S} d A\left(2 \mathcal{R}-K^{2}\right)
$$

which is a combination of the Hawking mass and the area [26]. The quantity $\mathcal{C}(S)$ has been used to good effect by Geroch, Jang and Wald [21, 22] in their approach to positive mass theorem and the Penrose inequality. In fact their work forms the base for recent progress [20] on the Riemannian Penrose inequality. We will see that $\mathcal{C}(S)$ tends to zero as $S$ tends to a round sphere of infinitesimal radius and also as $S$ tends to an asymptotic round sphere. 


\section{SPHERICAL SYMMETRY}

The Ricci flow is a tensor evolution equation and therefore commutes with diffeomorphisms. It follows that isometry groups are preserved under the Ricci flow. One way to approach the Ricci flow is to start with symmetric situations so that the complexity of the flow is reduced. If we impose so much symmetry that the spaces of interest are homoge-

neous, the RF becomes an ODE rather than a PDE. Such situations have been studied[24]. However, this assumption is too restrictive from our present physical motivation. We would like to deal with asymptotically flat (or AdS ) spaces since energy is defined with respect to an asymptotic structure. Homogeneous spaces which are asymptotically flat would be everywhere flat and not very interesting. Spherical symmetry has the advantage that there are non trivial asymptotically flat spaces. The Ricci flow reduces to a PDE with just two independent variables, the $\tau$ and $r$ co-ordinates. As we will see, spherically symmetric spaces will provide us with an analytical as well as numerical testing ground and pave the way for the general treatment. This special case is useful since one can develop a physical feel for the geometrical quantities of interest and easily produce physical examples and counterexamples as a guide to intuition.

While setting up the spherically symmetric initial data set, we will work with two forms of the metric. Each of these has its use and its limitation. We will refer to them as "a-form" and "b-form". They correspond to different choices of co-ordinate gauge.

- The a-form: In this form of the initial data set, the 3-metric is taken as

$$
d s^{2}=a(r) d r^{2}+r^{2}\left(d \theta^{2}+\sin ^{2} \theta d \phi^{2}\right)
$$

With this form of the metric we calculate the Ricci tensor and the scalar curvature: The nonzero components of the Ricci tensors are (a prime means differentiation with respect to $r$ )

$$
\begin{gathered}
R_{r r}=\frac{a^{\prime}}{r a}, \\
R_{\theta \theta}=\frac{a^{\prime} r}{2 a^{2}}+1-\frac{1}{a}, \\
R_{\phi \phi}=\sin ^{2} \theta\left(R_{\theta \theta}\right) .
\end{gathered}
$$

The scalar curvature is

$$
R=\frac{2}{r^{2}}+\frac{2 a^{\prime}}{r a^{2}}-\frac{2}{a r^{2}}
$$


The a-form is useful for the study of the evolution of Hawking mass $\mathcal{M}_{H}$. However, this form of the metric is not useful if there is an apparent horizon because in that case $a(r)$ blows up at the apparent horizon. To study the evolution of the area of the apparent horizon under RF we use the "b-form" of the metric discussed next.

- The b-form: In this form of the initial data set, the 3-metric is taken as

$$
d s^{2}=d r^{2}+b(r)\left(d \theta^{2}+\sin ^{2} \theta d \phi^{2}\right)
$$

With this metric we again calculate the Ricci tensor and the scalar curvature: The nonzero components of the Ricci tensor are

$$
\begin{gathered}
R_{r r}=\frac{b^{2}-2 b b^{\prime \prime}}{2 b^{2}}, \\
R_{\theta \theta}=1-\frac{b^{\prime \prime}}{2}, \\
R_{\phi \phi}=\sin ^{2} \theta\left(R_{\theta \theta}\right) .
\end{gathered}
$$

The scalar curvature is

$$
R=\frac{b^{\prime 2}-4 b\left(b^{\prime \prime}-1\right)}{2 b^{2}}
$$

a-form and Hawking Mass: Let us start with the a-form (11) of the metric and evaluate the Hawking mass functional (9) for $S$ chosen to be a sphere $r=$ constant. For this spherical topology, the first term in (10) gives $16 \pi$ by the Gauss-Bonnet theorem and

$$
\mathcal{M}_{H}(S):=\frac{\sqrt{\mathcal{A}}}{64 \pi^{\frac{3}{2}}}\left(16 \pi-\int_{S} K^{2} d A\right) .
$$

Let $\hat{n}_{a}$ be a unit normal to the surface $S$. The normalization

$$
h^{a b} \hat{n}_{a} \hat{n}_{b}=a^{-1}(r) \hat{n}_{r} \hat{n}_{r}=1
$$

fixes

$$
\hat{n}_{r}=\sqrt{a}
$$

and so

$$
\hat{n}^{a}=\left(\frac{1}{\sqrt{a}}, 0,0\right) .
$$

The trace of the extrinsic curvature is

$$
K=D_{a} \hat{n}^{a}=\frac{2}{r \sqrt{a}},
$$


so we have

$$
\int K^{2} d A=\frac{16 \pi}{a(r)}
$$

and

$$
\mathcal{M}_{H}(S)=\frac{\sqrt{\mathcal{A}}}{4 \pi^{1 / 2}}\left[1-\frac{1}{a(r)}\right]
$$

For flat space $a(r)=1$ and we have $M_{H}(S)=0$ for round spheres in flat space as expected. If we take the example of the exterior Schwarzschild space

$$
a(r)=\left(1-\frac{2 M}{r}\right)^{-1}
$$

and then for any $r>2 M$,

$$
M_{H}(S)=\frac{\sqrt{4 \pi r^{2}}}{4 \sqrt{\pi}}\left[1-\left(1-\frac{2 M}{r}\right)\right]=M
$$

For a general a-form metric we can write $a(r)$ as $\left(1-\frac{2 M(r)}{r}\right)^{-1}$. We find that [25]

$$
\mathcal{M}_{H}(S)=M(r)
$$

and the "compactness" works out to

$$
C(r)=32 \pi \frac{M(r)}{r}
$$

In order to get a better physical feel for what these geometrical quantities mean, let us consider some typical distributions of matter. Let us choose the matter density $\rho(r)=$ $(1 / 16 \pi) R(r)$ positive and plot the functions $\rho(r), C(r)$ and $M_{H}(r)$. Figure(10) displays the forms of these functions (in arbitrary units) for a spherical shell of matter. $C(r)$ increases to a maximum value and then decreases to 0 at infinity. For a matter distribution of two momentarily static shells of matter, the slightly more complex behaviour of $C(r)$ is shown in Fig. (2). For a star $C(r)$ attains its maximum near the surface of the star. From (24), in the Newtonian limit $C(r)$ is a constant times the dimensionless Newtonian potential, or the mass to radius ratio. Hence the name "compactness" is justified.

Note that $M_{H}(r)$ monotonically increases with $r$ to attain its asymptotic value. This is due to the local energy condition, which implies positive scalar curvature $R \geq 0$. This condition is conveniently stated in terms of the function $M(r)$. The scalar curvature of (11) is given by $R=4 M^{\prime}(r) / r^{2}$ and so the constraint of positivity of scalar curvature simply states that $M(r)$ is a non-decreasing function of $r$. Assuming that the form (11) 
holds all the way to the origin and that the scalar curvature $R$ is finite, we have $M(0)=$ $0, M^{\prime}(0)=0, M^{\prime \prime}=0, M^{\prime \prime \prime}(0) \geq 0 . M(r)$ increases from zero and tends to an asymptotic value $M_{A D M}=\lim _{r \rightarrow>\infty} M(r)$ which is the ADM mass of the metric. In a sense[25], $\mathcal{M}_{H}(r)$ measures the total mass contained within a sphere of areal radius $r . \mathcal{M}_{H}(r)$ is non-negative for all $r$ and and from (20), we conclude that $a(r) \geq 1$ for all $r$.

Note that from (19) it follows that if the space contains an apparent horizon $(K=0)$, $a(r)$ must diverge. This is why the a-form is unsuitable for treating apparent horizons. The b-form does not suffer from this problem.

Geometric quantities in the b-form: We now consider a round sphere $S \subset \Sigma$ given by $r=$ constant in the spherically symmetric "b-form" (12) of the metric. The unit normal satisfies

$$
h^{a b} \hat{n}_{a} \hat{n}_{b}=\hat{n}_{r} \hat{n}_{r}=1
$$

and $\hat{n}^{r}=(1,0,0)$. The area $\mathcal{A}$ of $S$ is given as

$$
\mathcal{A}(r)=\int_{S} \sqrt{\gamma} d \theta d \phi=4 \pi b(r),
$$

where $\gamma=b^{2} \sin ^{2} \theta$ is the determinant of the induced metric $\gamma_{i j}$ on $S$. The trace of the extrinsic curvature is given by

$$
K:=D_{a} \hat{n}^{a}=\frac{b^{\prime}}{b},
$$

(where a prime indicates differentiation with respect to $r$ ). The general formula for the compactness reduces in spherical symmetry to,

$$
C(r)=16 \pi-\int_{S} \sqrt{\gamma} d \theta d \phi K^{2}=16 \pi-\frac{4 \pi b^{2}}{b}
$$

and the Hawking mass is

$$
\mathcal{M}_{H}(r)=\frac{\sqrt{\mathcal{A}(r)}}{64 \pi^{3 / 2}} C(r)=\frac{\sqrt{b}}{2}\left[1-\frac{b^{\prime 2}}{4 b}\right] .
$$

Note that $\mathcal{M}_{H}$ depends on $b(r)$ and its derivative $b^{\prime}(r)$, in constrast to the simple algebraic relation (201) we had in the a-form of the metric.

Area under Ricci flow: We consider a metric initially in the b-form, evolving under a pure Ricci flow (without a diffeomorphism term). Under this evolution, the b-form may not be preserved. We view $S$ as a fixed surface of $\Sigma$ and so the co-ordinate location of the surface $S$ does not change and hence $\frac{d r}{d \tau}=0$. From the Ricci flow we have

$$
\frac{\partial h_{\theta \theta}}{\partial \tau}=-2 R_{\theta \theta}
$$


from which follows the formula

$$
\frac{d \mathcal{A}}{d \tau}=4 \pi \frac{\partial b}{\partial \tau}=-4 \pi\left(2-b^{\prime \prime}\right)
$$

for the instantaneous rate of change of area of $S$.

Using the scalar curvature $R$ for the "b-form" of the metric (13) we see that, in spherical symmetry,

$$
\frac{d \mathcal{A}}{d \tau}=-\frac{1}{2} \int_{S} \sqrt{\gamma} d \theta d \phi R-\frac{1}{4} C
$$

and so we arrive at the inequality (since $R \geq 0$ )

$$
\frac{d \mathcal{A}}{d \tau} \leq-\frac{1}{4} C .
$$

In the case of the Schwarzschild space, $R=0$ and so the first integral in (30) vanishes and we have

$$
\frac{d \mathcal{A}}{d \tau}=-\frac{1}{4} C(S) .
$$

Thus Schwarzschild space saturates our inequality (31), just as it saturates the Penrose inequality.

Area of apparent horizons under Ricci Flow: Let $S$ be a minimal surface (or apparent horizon, they coincide in the case of time symmetric data) in $\Sigma$ i.e $S$ is a closed two manifold embedded in $\Sigma$ with the property that the trace of the extrinsic curvature vanishes. We want to see how the area of $S$ varies under the RF. We start with the spherically symmetric "bform" of the metric (12). Let the location of the apparent horizon be at $r=r_{0}$. From (27), we have that $K=b^{\prime} /\left.b\right|_{r_{0}}=0$ The condition that the surface $r=r_{0}$ be an apparent horizon is

$$
\left.b^{\prime}\right|_{r=r_{0}}=0 .
$$

Can a minimal surface spontaneously appear if none was present initially? The answer is no, as the following argument shows by contradiction. Let us consider the $b$ form of the metric and evolve it by the Ricci flow supplemented by a suitable radial diffeomorphism $\xi^{a}$ chosen to maintain the $b$-form

$$
\begin{gathered}
\frac{\partial h_{r r}}{\partial \tau}=-2 R_{r r}+2(D \xi)_{r r}=0, \\
\frac{\partial h_{\theta \theta}}{\partial \tau}=\frac{\partial b}{\partial \tau}=-2 R_{\theta \theta}+2(D \xi)_{\theta \theta} .
\end{gathered}
$$


We may write $\xi_{r}=\partial_{r} f$ and using (34) and choose $f$ so that

$$
f^{\prime \prime}=\frac{b^{2}-2 b b^{\prime \prime}}{2 b^{2}} \text {. }
$$

(This leaves some freedom in $f$, but this does not affect the following.) (35) then gives us the evolution equation for $b(r, \tau)$ in the $b$ form of the metric

$$
\frac{\partial b}{\partial \tau}=b^{\prime \prime}-2+b^{\prime} f^{\prime}
$$

Differentiating this equation we arrive at an evolution equation for $b^{\prime}$

$$
\frac{\partial b^{\prime}}{\partial \tau}=b^{\prime \prime \prime}+b^{\prime \prime} f^{\prime}+b^{\prime} f^{\prime \prime},
$$

which gives us a maximum principle for $b^{\prime}$. Suppose that $b^{\prime}>0$ for all $\tau<\tau_{0}$ and that for the first time $\tau=\tau_{0}$, a minimal surface appears $\left(b^{\prime}\left(r_{0}\right)=0\right)$ at $r=r_{0}$. We have $\frac{\partial b^{\prime}}{\partial \tau}\left(r_{0}\right)<0$, since $b^{\prime}$ decreased to zero. On the other hand, since $r_{0}$ is a minimum of $b^{\prime}$, we have $b^{\prime \prime}\left(r_{0}\right)=0$ and $b^{\prime \prime \prime}\left(r_{0}\right) \geq 0$. A glance at (36) shows that $\frac{\partial b^{\prime}}{\partial \tau} \geq 0$, which is a contradiction. Thus a minimal surface cannot spontaneously appear under RF if it was not initially present.

Regions where $b^{\prime}<0$ are called trapped regions. Since the RF is continuous, such regions evolve continuously. Trapped regions are bounded by minimal surfaces $\left(b^{\prime}=0\right)$. From the last paragraph it is clear that trapped regions cannot spontaneously appear under RF since they are accompanied by minimal surfaces. However, trapped regions can continuously shrink to zero and disappear. When this happens, the two minimal surfaces that form the boundary of the trapped region merge and disappear. More descriptively, a minimal surface $\left(b^{\prime}=0, \quad b^{\prime \prime}>0\right)$ merges with a maximal surface $\left(b^{\prime}=0, \quad b^{\prime \prime}<0\right)$ and disappears.

Setting aside such mergers, let us study how the area of minimal surfaces evolves under the pure RF (1). During the RF the metric changes and the location of the horizon may change and so $r_{0}=r_{0}(\tau)$ where $\tau$ is the parameter of the RF. Also the geometry of $S$ will change. In principle both these effects could lead to change of area. The area is given by (26) $\mathcal{A}(r)=4 \pi b(r)$. The total rate of change of area, therefore, is

$$
4 \pi \frac{d b}{d \tau}=4 \pi\left[\left.\frac{\partial b}{\partial r}\right|_{r=r_{0}} \frac{d r_{0}}{d \tau}+\left.\frac{\partial b}{\partial \tau}\right|_{r=r_{0}}\right] .
$$

The first term vanishes because of the apparent horizon condition (33). The second term is evaluated by specialising eq.(31) to an apparent horizon. So the area $\mathcal{A}=4 \pi b$ satisfies

$$
\frac{\partial \mathcal{A}}{\partial \tau} \leq-4 \pi
$$


This implies that the area of the horizon is decreasing at least linearly with $\tau$. Since the area was finite to begin with, we find that (if the horizon persists) $b$ evaluated at the horizon goes to zero in a finite $\tau$.

Next we see that as $b \rightarrow 0$ we approach a singularity. To show this we will suppose that $R$ is finite and will arrive at a contradiction. As $b \rightarrow 0$ the scalar curvature

$$
R=\frac{2\left(1-b^{\prime \prime}\right)}{b}
$$

is finite only if $b^{\prime \prime}=1$. We Taylor expand in powers of $r-r_{0}$ about $r_{0}$, the location of the apparent horizon

$$
b(r)=b\left(r_{0}\right)+b^{\prime}\left(r_{0}\right)\left(r-r_{0}\right)+\frac{1}{2} b^{\prime \prime}\left(r_{0}\right)\left(r-r_{0}\right)^{2}+\ldots
$$

As $b\left(r_{0}\right) \rightarrow 0, b^{\prime \prime}\left(r_{0}\right) \rightarrow 1$ and $b^{\prime}\left(r_{0}\right)=0$ due to the apparent horizon condition (33), we have

$$
b(r)=\frac{1}{2}\left(r-r_{0}\right)^{2}
$$

so the metric is

$$
d s^{2}=d r^{2}+\frac{1}{2}\left(r-r_{0}\right)^{2}\left(d \theta^{2}+\sin ^{2} \theta d \phi^{2}\right) .
$$

Shifting the $r$ co-ordinate $r \rightarrow\left(r-r_{0}\right)$ gives the form

$$
d s^{2}=d r^{2}+\frac{1}{2} r^{2}\left(d \theta^{2}+\sin ^{2} \theta d \phi^{2}\right)
$$

So the volume of a ball of radius $r$ is

$$
\int d \theta d \phi d r \frac{\sqrt{r^{4} \sin ^{2} \theta}}{4}=\frac{1}{4}\left(\frac{4 \pi r^{3}}{3}\right) .
$$

We then find from the expression for the volume of a ball of radius $r$ (as $r \rightarrow 0$ ) centered at point $p$ that

$$
\text { volume } \mathcal{B}(p, r)=\left(\frac{4 \pi}{3}\right)\left(r^{3}-\frac{1}{30} R(p) r^{5}\right)
$$

and so the scalar curvature blows up, $R(p) \sim r^{-2}$, which is a contradiction to the assumption of finite $R$ that we started with. So an apparent horizon which persists under RF results in a singularity in a finite amount of $\tau$ parameter $\tau \leq \tau_{0}$, where $\tau_{0}=\mathcal{A}(0) /(4 \pi)$.

Compactness under RF: We have previously derived the relation (24) for the compactness of a sphere $r=$ const from which follows

$$
C(r)=16 \pi[1-1 / a]
$$


A calculation reveals that under $\mathrm{RF}$

$$
\frac{d C}{d \tau}=-\frac{2 M^{\prime}(r)}{r^{2}}+\frac{2 M^{\prime \prime}}{r} .
$$

While the first term here is of definite sign, the second term is not. As a result, the rate of change of $C$ with $\tau$ is not monotonic. Note however that for Schwarzschild $M(r)$ is a constant $M$ and so $d C / d \tau=0$.

The Hawking mass is given by $\mathcal{M}_{H}(r)=r C(r) /(32 \pi)$ and to work out its rate of change $\frac{d \mathcal{M}_{H}}{d \tau}$ under the RF, we need to find $d r / d \tau$. This is easily read off from (30) and we find

$$
\frac{d r}{d \tau}=\frac{-M^{\prime}}{r}-\frac{C}{32 \pi r} .
$$

Putting these equations together we can work out $\frac{d \mathcal{M}_{H}}{d \tau}$ and it turns out to be a linear combination of $M^{\prime}$ and $M^{\prime \prime}$. As a result, the Hawking mass is not monotonic along the pure Ricci flow in the a-form of the metric. It may be that adding a suitable diffeomorphism will result in monotonic behaviour for $\mathcal{M}_{H}$.

Maximum principle for compactness: However, we can make a diffeo invariant statement about the behaviour of compactness. We have already seen that if a minimal surface is initially present this may result in a finite $\tau$ singularity. Let us suppose that no minimal surface is initially present. None develops under RF, as we saw earlier. We can therefore use the a-form of the metric. We have seen that $C(r)$ starts from 0 at $r=0$, reaches a maximum (perhaps several local maxima) and then decays to zero as $r \rightarrow \infty$. Let us focus on $C_{\max }$ the value of $C$ at its absolute maximum. Just as for apparent horizons, the value of $C_{\max }$ is not affected by a diffeomorphism: moving the surface does not affect the value of $C_{\max }$ since we are at a maximum. We choose a diffeomorphism to preserve the a-form of the metric and then the evolution equation for $a(r)$ reads

$$
\frac{\partial a(r)}{\partial \tau}=\frac{a^{\prime \prime}(r)}{a(r)}-\frac{3\left(a^{\prime}(r)\right)^{2}}{2 a(r)^{2}}-\frac{2(a(r)-1)+r a^{\prime}(r)(1-a(r)) / a(r)}{r^{2}},
$$

where a prime denotes differentiation with respect to $r$. We have studied the evolution of (41) using numerical techniques. Some of the results presented here were initially suggested by the numerical evidence.

Let us focus on the maximum value of $a(r)$. Recall that $a(r) \geq 1$. At the maximum value of $a(r)$, we have $\left.a^{\prime}(r)\right|_{\max }=0$ and $\left.a^{\prime \prime}(r)\right|_{\max } \leq 0$. So from equation (41) we see that the 
maximum value of $a(r)$ is monotone non-increasing as the flow parameter $\tau$ increases i.e.,

$$
\frac{\partial a(r)_{\max }}{\partial \tau}=\frac{a^{\prime \prime}(r)}{a(r)}-\frac{2\left(a(r)_{\max }-1\right)}{r^{2}} \leq 0 .
$$

A maximum of $C$ corresponds via (38) to a maximum of $a(r)$. Clearly, a maximum principle for $a(r)$ implies a maximum principle for compactness $C(r)$ and we have the inequality

$$
\frac{d C(r)_{\max }}{d \tau} \leq 0
$$

We can use this maximum principle to comment on the long time existence of the spherically symmetric asymptotically flat Ricci flow. Let us suppose that no minimal surface is initially present. From (42), we see that for the LHS of (42) to vanish we must have $a(r)_{\max }=1$, which implies $a(r)=1$ identically. This describes flat space. If the initial metric is not flat space, its $a(r)_{\max }$ decreases with the flow and finally attains the flat space fixed point $a(r)=1$. Our argument shows clearly that in spherical symmetry, the only asymptotically flat fixed point of the flow is flat space. This recovers results obtained by other methods [7, 27].

To summarise, the maximum principle for compactness leads to a criterion for the existence of the Ricci flow in the asymptotically flat case. If there are no apparent horizons, the flow exists for all $\tau$ and converges to flat space. If there are apparent horizons, the Ricci flow either terminates in finite time singularity or removes the horizons by mergers.

\section{AREA UNDER RICCI FLOW}

We now drop the assumption of spherical symmetry and deal with a general asymptotically flat manifold $\Sigma$, with one end at infinity and a fixed closed orientable surface $S$ of arbitrary topology embedded in $\Sigma$. The induced metric of $S$ is written $\gamma_{i j}$ and extrinsic curvature tensor of $S$ is written $K_{i j}$, where $i, j$ are two dimensional indices in the space tangential to $S$. We will sometimes use projected $a, b$ indices for these. The trace of the extrinsic curvature is written $K=\gamma^{i j} K_{i j}$. As the metric $h_{a b}$ evolves according to the Ricci flow (11), how does the area change with $\tau$ ? Since the metric of $\Sigma$ is changing, we have to remember that the unit normal to $S$ is also changing with the metric.

Let us define the surface $S$ as the level set of a function $\eta$ on $\Sigma$ which is strictly increasing outward from $S$. Quite independent of any metric, the normal $\eta_{a}=D_{a} \eta$ is a well defined 
co-vector. $\eta_{a}$ is non-zero (since we assumed $\eta$ is not locally constant). (Choosing a different function $\eta$ will result in multiplication of $\eta_{a}$ by a positive function on $S$.) The unit normal

$$
\hat{n}_{a}=\frac{\eta_{a}}{(\eta \cdot \eta)^{1 / 2}}
$$

depends on the metric. As the metric changes the unit normal can only change by a multiple of itself $d \hat{n}_{a} / d t=\alpha \hat{n}_{a}$. By differentiating $\hat{n} . \hat{n}=1$, we arrive at

$$
\alpha=\frac{1}{2} \frac{d h^{a b}}{d \tau} \hat{n}_{a} \hat{n}_{b}
$$

(where in our notation, $\frac{d h^{a b}}{d \tau}$ is defined as $\frac{d h_{a b}}{d \tau}$ with its indices raised using the metric tensor $\left.h^{a b}\right)$.

Starting from the definition (8) we compute $\frac{d \mathcal{A}}{d \tau}$

$$
\frac{d \mathcal{A}}{d \tau}=\int_{S} d^{2} x \frac{d \sqrt{\gamma}}{d \tau}
$$

We easily see that

$$
\frac{d \sqrt{\gamma}}{d \tau}=\frac{1}{2} \sqrt{\gamma} \gamma^{a b} \frac{d h_{a b}}{d \tau}
$$

and we arrive at

$$
\frac{d \mathcal{A}}{d \tau}=\frac{1}{2} \int_{S} \sqrt{\gamma} d^{2} x\left(h^{a b}-\hat{n}^{a} \hat{n}^{b}\right) \frac{d h_{a b}}{d \tau} .
$$

Area under ricci flow: Using the form (11) of the Ricci flow, we find

$$
\frac{d \mathcal{A}}{d \tau}=\int_{S} \sqrt{\gamma} d^{2} x\left[\hat{n}^{a} \hat{n}^{b} R_{a b}-R\right] .
$$

From the Gauss-Codazzi equation[25], we have

$$
\hat{n}^{a} \hat{n}^{b} R_{a b}-R=-1 / 2\left[R+\mathcal{R}+\left(K_{i j} K^{i j}-K^{2}\right)\right]
$$

which can be rearranged to give

$$
\begin{aligned}
\frac{d \mathcal{A}}{d \tau} & =-1 / 2 \int_{S} d^{2} x \sqrt{\gamma}\left[R+\left(K^{i j}-\frac{1}{2} K \gamma^{i j}\right)\left(K_{i j}-\frac{1}{2} K \gamma_{i j}\right)\right] \\
& -\frac{1}{4} \int_{S} d^{2} x \sqrt{\gamma}\left(2 \mathcal{R}-K^{2}\right) .
\end{aligned}
$$

The second integral in (51) is identified as $-\mathcal{C}(S) / 4$, minus one fourth the compactness integral of $S$ and the first integral, which is of definite sign can be dropped to arrive at the inequality

$$
\frac{d \mathcal{A}}{d \tau} \leq-\frac{\mathcal{C}(S)}{4}
$$


This inequality is one of the main results of this paper. This result can be reexpressed in terms of the Hawking Mass:

$$
\frac{d \mathcal{A}^{3 / 2}}{d \tau} \leq-24 \pi^{3 / 2} \mathcal{M}_{H}(S)
$$

Thus the rate of decrease of area of a closed 2-surface under Ricci flow is bounded by the Hawking mass. As we mentioned earlier, the inequality (52) is saturated in the case of the spheres of Schwarzschild space (which is given by (111) with $a(r)=(1-2 M / r)^{-1}$ ). In this case $R=0$ and the spheres are shear free $\left(K_{i j}=\frac{1}{2} K \gamma_{i j}\right)$, so the first integral in (51) vanishes. We conjecture that round spheres in Schwarzschild are the only surfaces which saturate this bound.

As a simple application of this inequality, let us consider flat space. Since the Ricci tensor vanishes we have that $d \mathcal{A} / d \tau=0$ and so the LHS of (53) vanishes. We arrive at the conclusion that for all surfaces in flat space, the Hawking mass is non positive! This fact has also been noticed in [28], where a direct proof is given. In fact, the converse of this statement is also true: Given positive scalar curvature, flat space is the only one for which the Hawking mass is non-positive. To see this, note that the supremum over $S \in \Sigma$ of the Hawking mass is the ADM mass and if this supremum vanishes, it follows from the positive mass theorem that the space must be flat.

Area under diffeos: Under a diffeo, the metric changes as in (4) and so we have from (48)

$$
\frac{d \mathcal{A}}{d \tau}=\frac{1}{2} \int \sqrt{\gamma} d^{2} x\left(h^{a b}-\hat{n}^{a} \hat{n}^{b}\right) 2 D_{a} \xi_{b}=\int \sqrt{\gamma} d^{2} x\left[D_{a} \xi^{a}-n^{a} n^{b} D_{a} \xi_{b}\right]
$$

If we suppose that $\xi^{a}$ is tangent to $S$, then with $\tilde{D}_{a}$ denoting covariant derivative intrinsic to $\left(\gamma_{a b}, S\right)$,

$$
\tilde{D}_{a} \xi_{b}=\gamma_{a}^{a^{\prime}} \gamma_{b}^{b^{\prime}} D_{a^{\prime}} \xi_{b^{\prime}}
$$

gives us

$$
\tilde{D}_{a} \xi^{a}=\gamma^{a b} \tilde{D}_{a} \xi_{b}=\left(h^{a b}-\hat{n}^{a} \hat{n}^{b}\right) D_{a} \xi_{b}
$$

So, as one would expect,

$$
\frac{d \mathcal{A}}{d \tau}=\int \sqrt{\gamma} d^{2} x\left[\tilde{D}_{a} \xi^{a}\right]=0
$$

since this is a divergence over a closed surface $S$. It is therefore enough to consider the component of $\xi$ normal to $S$. Let

$$
\xi^{a}=u \hat{n}^{a} .
$$


Then

$$
\frac{d \mathcal{A}}{d \tau}=\int \sqrt{\gamma} d^{2} x\left(h^{a b}-\hat{n}^{a} \hat{n}^{b}\right) D_{a}\left(u \hat{n}_{b}\right)
$$

which works out to the standard answer

$$
\frac{d \mathcal{A}}{d \tau}=\int \sqrt{\gamma} d^{2} x u \gamma^{a b} D_{a} n_{b}=\int \sqrt{\gamma} d^{2} x u K
$$

Area of horizons under ricci flow: If $S$ is a minimal surface, we find from (10) that since $K=0$, the area changes according to

$$
\frac{d \mathcal{A}(S)}{d \tau} \leq \frac{1}{4} C(S)=-\frac{8 \pi \chi(S)}{4}
$$

where $\chi(S)$ is the Euler characteristic of $S$. For a minimal surface of spherical topology, we have

$$
\frac{d \mathcal{A}}{d \tau} \leq-4 \pi
$$

which we had seen earlier for the special case of spherical symmetry. This result is unaffected by adding a diffeo to the RF because of (60) and the minimal surface condition $K=0$.

An interesting special case is one for which $S$ is the outermost horizon with respect to asymptotic infinity. $\mathrm{S}$ is defined as the boundary of the region having trapped surfaces. $\mathrm{S}$ is a minimal surface and is known to have spherical topology. According to (62), the area of $S$ will shrink. Under the Ricci flow, the trapped region cannot disappear suddenly, but evolves continuously, because trapped surfaces remain trapped under small perturbations. Near $S$ there will be a new minimal surface with the same area. If the initial area is $\mathcal{A}_{0}$, it dissappears within a time $\mathcal{A}_{0} / 4 \pi$, either by merger or by shrinking to zero.

\section{HAWKING MASS UNDER RICCI FLOW}

The Hawking mass of a closed surface $S$ given by (9) is a combination of the "compactness" of $S$ and its area. To study the evolution of the Hawking mass, it is enough to understand the evolution of the compactness, the evolution of area being already treated in the last section. From the formula (10) for compactness, we see that the first term $\int d^{2} x \sqrt{\gamma} \mathcal{R}$ drops out on differentiation since it is a topological invariant by the Gauss-Bonnet theorem. The second term gives

$$
\frac{d C(S)}{d \tau}=-\frac{d}{d \tau} \int K^{2} \sqrt{\gamma} d^{2} x=-\int 2 K \sqrt{\gamma} \frac{d K}{d \tau} d^{2} x-\int K^{2} \frac{d \sqrt{\gamma}}{d \tau} d^{2} x
$$


Using (47) for the second term in (63) and the formula

$$
\frac{d K}{d \tau}=\frac{d}{d \tau}\left(D_{a} \hat{n}^{a}\right)=\frac{d \Gamma_{a m}^{a}}{d \tau} \hat{n}^{m}+D_{a} \frac{d \hat{n}^{a}}{d \tau},
$$

we find after a straightforward calculation that

$$
\begin{aligned}
\frac{d C}{d \tau} & =-\int d A K\left[h^{a b} \hat{n}^{c} D_{c} \frac{d h_{a b}}{d \tau}-2 D_{a}\left(\frac{d h^{a b}}{d \tau} \hat{n}^{b}\right)+\hat{n}^{a} D_{a}\left(\frac{d h^{c d}}{d \tau} \hat{n}_{c} \hat{n}_{d}\right)\right] \\
& -\int d A \frac{K^{2}}{2}\left[\frac{d h^{c d}}{d \tau} \hat{n}_{c} \hat{n}_{d}+h^{a b} \frac{d h^{a b}}{d \tau}\right] .
\end{aligned}
$$

Equations (66) give the general evolution of the compactness under for any one parameter family $h_{a b}(\tau)$ of metrics. We will specialise these equations to the two cases of interest: the pure Ricci flow and a pure diffeo.

Compactness under ricci flow: Substituting $\frac{d h_{a b}}{d \tau}=-2 R_{a b}$ into (66) we find after simplification using the contracted Bianchi identity that

$$
\frac{d C}{d \tau}=\int_{S} d A\left\{K^{2}\left(R+\hat{n}^{a} \hat{n}^{b} R_{a b}\right)-2 K\left[2 R^{a b} D_{a} \hat{n}_{b}-\hat{n}^{a} D_{a}\left(R^{c d} \hat{n}_{c} \hat{n}_{d}\right)\right]\right\} .
$$

Compactness under diffeos: How does the compactness change under a pure diffeo generated by $\xi^{a}$ ? As we saw for the area, the tangential component of $\xi^{a}$ does not cause any change in the integral. The diffeo vector field can be characterised by its normal component $\xi^{a}=u \hat{n}^{a}$. Substituting (44) into (66) and simplifying and using the Gauss-Codazzi equation [25]

$$
-2 n^{a} n^{b} R_{a b}+R=\mathcal{R}+\left(k^{i j} k_{i j}-k^{2}\right)
$$

gives us the formula [22]

$$
\frac{d C}{d \tau}=\int_{S}\left[2 K \tilde{D}^{a} \tilde{D}_{a} u+u K \sigma^{i j} \sigma_{i j}+u k R-\frac{1}{2} u K\left(2 \mathcal{R}-K^{2}\right)\right] \sqrt{\gamma} d^{2} x
$$

where $\tilde{D}_{a}$ denotes the intrinsic covariant derivative operator within the surface and $\sigma^{i j}=$ $K^{i j}-1 / 2 \gamma^{i j} K$. If there exists a diffeo such that $u K=1$ (this is the inverse mean curvature flow (IMC)) one can conclude [22] that under this diffeo

$$
\frac{d C}{d \tau} \geq-C / 2
$$

This inequality (which too is saturated by the Schwarzschild space) was used by Jang and Wald[21] to prove the Penrose inequality. We can get an expression for the evolution of Hawking mass. The Hawking mass is

$$
\mathcal{M}_{H}(S)=\frac{\sqrt{\mathcal{A}(S)}}{64 \pi^{3 / 2}} C(S)
$$


We then have

$$
\frac{d}{d \tau} \mathcal{M}_{H}=\left(\frac{1}{64 \pi^{3 / 2}}\right)\left(\frac{1}{2 \sqrt{\mathcal{A}}} \frac{d \mathcal{A}}{d \tau} C+\sqrt{\mathcal{A}} \frac{d C}{d \tau}\right)
$$

Knowing $\frac{d \mathcal{A}}{d \tau}$ and $\frac{d C}{d \tau}$ for a flow, can calculate $\frac{d}{d \tau} \mathcal{M}_{H}$. (60) and Geroch's inequality (70) then imply that the Hawking mass is monotonic under the IMC flow.

A maximum principle for compactness? The compactness $C(S)$ of a closed surface $S$ tends to zero for $S$ tending to a small round sphere, and also tends to zero for a round spheres at asymptotic infinity. We would expect based on our experience with spherical symmetry, that somewhere in between, there is a surface $S$ for which $C(S)$ attains its global maximum. If this maximum is equal to $16 \pi$, then $S$ is a minimal surface and conversely. If the maximal compactness is less than $16 \pi$, we may expect from the spherically symmetric case that this value $\left.C(S)\right|_{\max }$ will monotonically decrease to zero.

$$
\frac{d C_{\max }}{d \tau} \leq 0
$$

with the equality holding only for flat space. A zero value for $\left.C(S)\right|_{\max }$ would also imply a non-positive value for $\mathcal{M}_{H}(S)$. Supremising this over $S$ tells us that the ADM mass of the space vanishes, which implies (by the positive energy theorem[29]) that space is flat. We conjecture that (73) is true generally, but have not been able to establish the truth of this conjecture. If this conjecture is true, it would imply that in the long time, an initial metric without minimal surfaces would approach flat space.

\section{CONCLUSION}

We have described some applications of Ricci flow techniques to asymptotically flat spaces in general relativity. Our main result is that under the Ricci flow, the rate of change of area of a closed surface is bounded by its Hawking mass. This inequality is saturated by the Schwarzshild space. Since the Schwarzschild space saturates the Penrose inequality as well as Geroch's (70), our inequality may be related to these. We have also studied the behaviour of compactness under the Ricci flow. Our work in spherical symmetry suggests that there may be a maximum principle for the compactness. The compactness is a functional on closed two dimensional surfaces. The conjecture is that for positive scalar curvature, the maximum value of this functional decreases under the Ricci flow plus diffeomorphisms. This proposal is a functional maximum principle unlike the more usual ones which are which are 
formulated on functions. If such a principle does exist, it would be of great interest as a diagnostic for the long term existence of the asymptotically flat Ricci flow. Most of the mathematical work in the area of Ricci flows is concerned with closed three manifolds. One of our main points here is that there may be interesting physical applications of these ideas to asymptotically flat spaces. For instance, [5] poses the question of stability of Euclidean flat space $\mathbb{R}^{3}$ under RF. If a maximum principle for compactness exists, it would imply that flat space is indeed stable under RF.

In general a minimal surface can have any topology. Special interest attaches to the case where $S$ is an outermost horizon. In this case one can physically identify $S$ as the boundary of a black hole region. Outermost horizons have the property that the surface has minimum area (and not just stationary area). This immediately implies [30] (when curvature is positive) that the outermost horizon has spherical topology. Our general result shows that outermost horizons always shrink under the Ricci flow. This result is diffeomorphism invariant. If one identifies the area of an outermost horizon as black hole entropy, we arrive at the conclusion that entropy is monotonically decreasing along the RF. A similar conclusion has been reached by [11] in a slightly different context: entanglement entropy of a two dimensional black hole is monotonically decreasing along the RF.

If one uses the Ricci flow to model the approach of a system to thermal equilibrium, one would expect that the entropy increases along the flow. However, we have seen that outermost horizons have spherical topology and their area decreases under the flow. In fact, it has been observed [12] that while the Ricci flow does have something in common (memory loss) with approach to equilibrium, the analogy is not perfect. A slight modification [12] of the Ricci flow is necessary in order for the entropy to be identified with black hole entropy. It may be that such a modification of the RF is necessary for application to black hole physics.

This paper was concerned exclusively with the case of asymptotically flat spaces. It would be interesting to generalise the treatment to allow for asymptotically AdS spaces. This appears to be a straightforward generalisation and some of the necessary changes are mentioned in [12, 30]. 


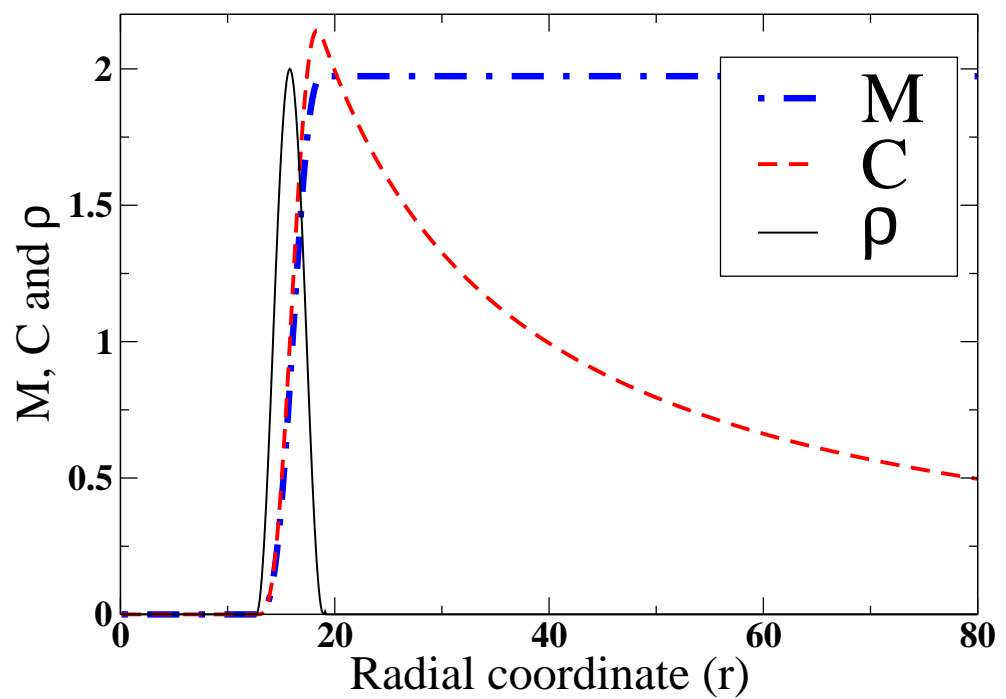

FIG. 1: Note that $\mathcal{M}_{H}(r)$ increases with $r$ to attain its asymptotic ADM value. But $C(r)$ increases to a maximum value and then decreases to 0 at infinity.

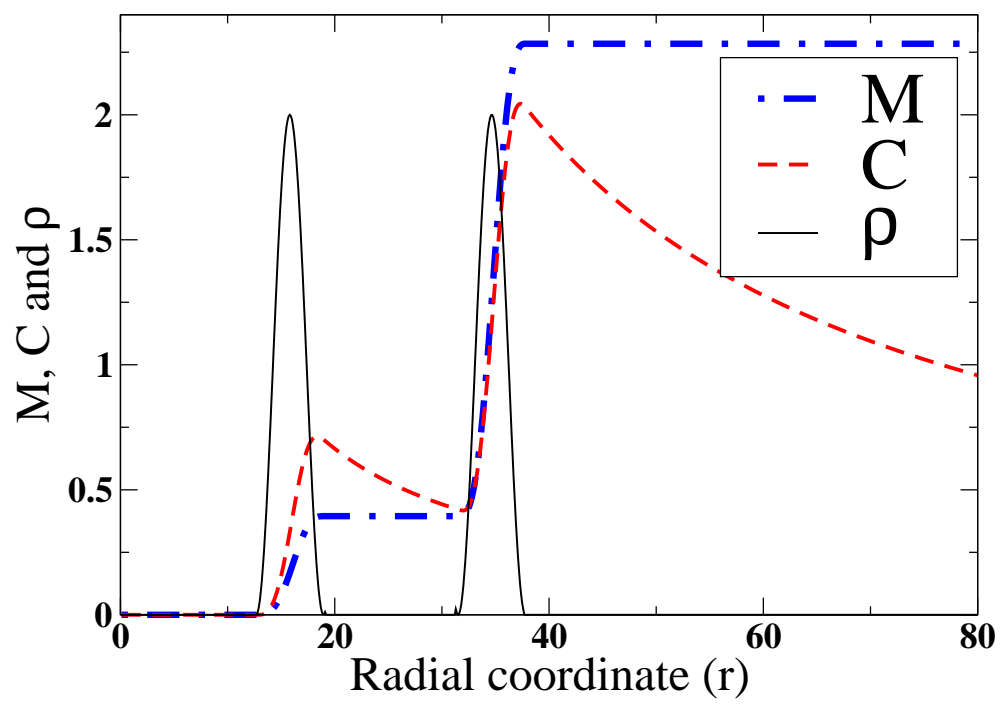

FIG. 2: Two shells of matter. 


\section{Acknowledgements:}

We thank Javed Ahmad for his collaboration and Harish-Chandra Research Institute for their hospitality in the early stages of this work. One of us (JS) thanks Harish Seshadri for discussions on Ricci flows.

\section{References}

[1] Friedan D 1980 Phys. Rev. Lett. 451057

[2] Hamilton R S 1982 Three-manifolds with positive Ricci curvature J. Differential Geom. 17

[3] Perelman G 2002 The entropy formula for the Ricci flow and its geometric applications Preprint math.DG/0211159

[4] Cao H-D and Chow B 1999 Recent developments on the Ricci flow Bull. Amer. Math. Soc. 36 59-74

[5] Woolgar E "Some Applications of Ricci Flow in Physics" eprint no arXiv:0708.2144

[6] Oliynyk T, Suneeta V and Woolgar E "A Metric for Gradient RG Flow of the Worldsheet Sigma Model" eprint: arXiv:0705.0827 to be published (Phys. Rev. D.)

[7] Oliynyk T and Woolgar E "Asymptotically Flat Ricci Flows" arXiv:math/0607438

[8] Oliynyk T, Suneeta V and Woolgar E 2006 " A gradient flow for world sheet non linear sigma models" Nucl. Phys.B. 739 441-458

[9] Oliynyk T, Suneeta V and Woolgar E 2005 "Irreversibility of world sheet renormalisation group flow" Phys.Lett. B. $\mathbf{6 1 0}$ 115-121

[10] Headrick M and Wiseman T 2006 Class.Quant.Grav. 23 6683-6708

[11] Soludukhin S 2007 Phys.Lett. B. 646 268-274

[12] Samuel J and Chowdhury S R 2007 Geometric Flows and Black Hole Entropy Class. Quantum Grav. 24 F1-F8

[13] Bekenstein J D 1994 Phys. Rev. D. 9 3292; Bekenstein J D 1981 Phys. Rev. D. 23287

[14] Bousso R “A Covariant Entropy Conjecture" 1999 JHEP 9907 004, arXiv:hep-th/9905177; Bousso R "The Holographic Principle” 2002 Rev.Mod.Phys. 74 825, arXiv:hep-th/0203101

[15] Unruh WG and Wald RM 1983 Phys. Rev. D 27, 2271 - 2276 
[16] Penrose R 1973 Ann. NY Acad. of Science 224

[17] Wald R M 1984 General Relativity University of Chicago Press

[18] Bray H L 2003 Preprint math.DG/0304261

[19] Bray H L 2001 J. Diff. Geom. 59 177-267

[20] Huisken G and Ilmanen T 2001 J. Differential Geom. 59 no.3

[21] Jang P S and Wald R M 1977 J. Math. Phys. 1841

[22] Geroch R 1973 Energy Extraction Ann. NY Acad. of Science 224

[23] Topping P 2006 London Mathematical Society Lecture Notes Series Cambridge University Press

[24] Chow B and Knopf D 2004 The Ricci Flow: An Introduction American Mathematical Society

[25] Poisson E 2004 A Relativist's Toolkit Cambridge Univ. Press

[26] We use the word compact not in the mathematical sense, but in the physical sense as in "A neutron star is a compact object".

[27] Ivey TA "The Ricci flow on radially symmetric $\mathbb{R}^{3}$ " 1994 Comm. Partial Differential eqns. 1481

[28] Hayward S A 1994 Phys. Rev. D. 49831

[29] Schoen R and Yau S-T 1979 Positivity of the Total Mass of a General Space-Time Phys. Rev. Lett. 4320

[30] Gibbons G W 1999 Class. Quant. Grav. 161677 Gibbons G W 1996 Tunnelling with a negative cosmological constant Nucl. Phys. B472 683-708 Gibbons G W 1998 Class. Quantum Grav. 15 2605-2612 